\title{
Cancer treatment associated cardiac toxicities
}

\author{
Eric Y. Chang*, Aneese F. Chaudhry, Victoria Lopez, Sanjay R. Jain
}

Department of Medicine, Morehouse School of Medicine, Atlanta, GA, USA

Received: 04 January 2018

Accepted: 29 January 2018

\section{*Correspondence to:}

Dr. Eric Y. Chang,

Email: eric.young.chang@

gmail.com

Copyright: (C) the author(s), publisher and licensee Medip Academy. This is an openaccess article distributed under the terms of the Creative Commons Attribution NonCommercial License, which permits unrestricted noncommercial use, distribution, and reproduction in any medium, provided the original work is properly cited.

\begin{abstract}
Cardiovascular disease remains the leading cause of mortality and morbidity in men and women both in the US and worldwide. With increased access to healthcare, it is predicted that life expectancies in developed countries will continue to rise and thus, lead to an increase in both cardiovascular disease and cancer. Similarly, improved survival rates in cancer patients have led to an increased awareness of the presence and potential worsening of cardiovascular disease in these patients. Cardiovascular complications due to side effects from cancer therapy or from cancer progression can be a common occurrence. Although recent advances in cancer therapeutics have led to improved survival rates and quality of life, the increase in life expectancy may be counteracted by the increased morbidity and mortality from progressive cardiac pathology. Examples of such complications include local invasion or distant metastatic spread, which can lead to superior vena cava syndrome, cardiac tamponade, or hyperviscosity syndromes. In addition, many chemo and radiation therapies can be directly toxic to the cardiovascular system. This review aims to discuss the potential cardiac toxicities of the most commonly used chemotherapeutics along with some strategies to manage these complex patients.
\end{abstract}

Keywords: Cardiac toxicity, Chemotherapy, Cancer

\section{INTRODUCTION}

Cardiovascular disease remains the leading cause of mortality and morbidity in men and women both in the US and worldwide. ${ }^{1,2}$ With increased access to healthcare, it is predicted that life expectancies in developed countries will continue to rise and thus, lead to an increase in both cardiovascular disease and cancer. Similarly, improved survival rates in cancer patients have led to an increased awareness of the presence and potential worsening of cardiovascular disease in these patients. Cardiovascular complications due to side effects from cancer therapy or from cancer progression can be a common occurrence. ${ }^{1,2}$ Although recent advances in cancer therapeutics have led to improved survival rates and quality of life, the increase in life expectancy may be counteracted by the increased morbidity and mortality from progressive cardiac pathology. Examples of such complications include local invasion or distant metastatic spread, which can lead to superior vena cava syndrome, cardiac tamponade, or hyperviscosity syndromes. In addition, many chemo and radiation therapies can be directly toxic to the cardiovascular system. This review aims to discuss the potential cardiac toxicities of the most commonly used chemotherapeutics along with some strategies to manage these complex patients.

\section{CARDIAC TOXICITY RELATED TO CANCER THERAPY: DEFINITIONS}

The definition of cancer therapy induced cardiotoxicity remains controversial with no consensus on official criteria. One classification that is used is the definition provided by the Cardiac Review and Evaluation 
Committee (CREC) who studied cardiac dysfunction rates for patients receiving trastuzumab. ${ }^{3}$

CREC's criteria for cardiac dysfunction included the presence of any of the following:

1. Cardiomyopathy characterized by the decrease in cardiac left ventricular ejection fraction (LVEF) that was either global or more severe in the septum;

2. Symptoms of congestive heart failure $(\mathrm{CHF})$;

3. Associated signs of CHF, including but not limited to S3 gallop, tachycardia, or both;

4. Decline in LVEF of at least $5 \%$ to $<55 \%$ with accompanying signs or symptoms of $\mathrm{CHF}$, or a decline in LVEF of at least $10 \%$ to $<55 \%$ without accompanying signs or symptoms.

The American College of Cardiology and the American Heart Association classification of heart failure patients emphasizes development and progression of the disease by delineation into stages.

- $\quad$ Stage A patients are targeted to reduce risk factors that could lead to heart failure.

- $\quad$ Stage B patients have asymptomatic heart failure and are prescribed beta blockers and ACE-inhibitors to prevent further left ventricular remodeling.

- Stage C patients are provided diuretics, digoxin, and/or aldosterone antagonists in addition to therapies mentioned above to further manage symptoms.

- $\quad$ Stage D patients have severe heart failure that is refractory to treatment. These patients may need to be evaluated for more invasive cardiac interventions.

The New York Heart Association (NYHA) provides an alternative classification of heart failure, categorizing patients according to limitations on physical activity.

- Class I patients generally do not have any restrictions with normal every day physical activity. These patients should participate in lifestyle modifications to reduce risk factors (proper diet, exercise, smoking cessation etc.).

- Class II patients may have slight restrictions with normal every day activities and will benefit from beta blockers and ace inhibitors.

- $\quad$ Class III patient experience definite limitations with activity with dyspnea on exertion and orthopnea. These patients will benefit from dietary modifications and diuretics (in addition to the medications listed above) to control fluid retention and overload.

- $\quad$ Patients who are Class IV have significant symptoms even at rest and will require more invasive intervention in addition to the medical therapies described above.

\section{Cancer therapy: associated cardiac toxicity}

Cardiotoxicity from chemotherapy is determined by numerous factors that depend on both the characteristics of the patient and the chemotherapy itself. Some patient factors include age, underlying disease, previous history of cardiovascular disease, prior mediastinal radiation, and the presence of various modifiable cardiovascular risk factors such as hypertension, diabetes, obesity, and smoking status. ${ }^{4}$ Chemotherapeutic factors include the class of drug, potential drug-drug interactions, route of administration, cumulative dosage, and allergic effects. ${ }^{1-3}$ It is important for all cancer patients to be properly evaluated to assess for baseline cardiovascular function prior to initiation of therapy.

Evaluation of cardiovascular function and assessment of potential modifiable cardiac risk factors are an important aspect of cancer management and can be initiated in the primary care setting, beginning with a comprehensive clinical exam to assess baseline function such as exercise capacity and resting heart rate. ${ }^{5}$ Electrocardiograms (EKGs) can be useful in evaluating for conduction abnormalities, QT interval prolongations, and other underlying cardiac disease. Echocardiograms can determine baseline ejection fraction, systolic and diastolic cardiac dysfunction, and wall motion abnormalities, all of which may be worsened by chemotherapy and lead to arrhythmias and/or heart failure.

Cardiotoxicity as a result of chemotherapy can occur at any time during the course of treatment, with side effects ranging from asymptomatic presentation and transient cardiac abnormalities (i.e. arrhythmias, hyper or hypotension, left ventricular dysfunction, QT prolongation), to life-threatening acute coronary syndrome, acute decompensated heart failure, or even death. ${ }^{1-3}$ Since initial changes in cardiac function can be subclinical and can occur at any time, it is important to maintain chronic, close monitoring of cardiac function throughout the treatment process.

Cardiovascular morbidity and mortality depends on the class of agent involved in therapy. The three broad categories of agents that are implicated in cardiovascular toxicity are the anthracyclines, non-anthracyclines, and biological or targeted agents.

\section{Anthracyclines}

Anthracycline cancer chemotherapeutics include doxorubicin, daunorubicin, idarubicin, epirubicin, and mitoxantrone. Historically, these agents were limited by dose-dependent myelosuppression, which prevented use of high-dose therapy that could possibly lead to cardiotoxicity. Recent advancements in both chemotherapy and supportive management has led to utilization of higher doses, which has resulted in an increased risk of side effects. ${ }^{4,6}$ Currently, anthracyclines are the class of chemotherapeutic agents most commonly 
associated with cardiotoxicity. For example, patients who develop doxorubicin-induced heart failure have a mortality rate of $50 \%$ within two years. ${ }^{4,6}$

Doxorubicin interacts with DNA by binding to the enzyme Topoisomerase-II and stopping the replication of DNA. In the process, toxic free oxygen radicals are generated, leading to lipid peroxidation and destruction of cell membranes. ${ }^{7,8}$ Topoisomerase-II alpha is overexpressed in tumors, but its analog, Topoisomerase-II beta, is expressed in normal cardiac myocytes. Binding of doxorubicin to this subset of enzymes leads to destruction of cardiomyocytes. ${ }^{9}$

In addition, multiple studies have shown that doxorubicininduced cardiotoxicity may be a multifactorial event. Other potential mechanisms of action include decreased adenosine triphosphate (ATP) production, mitochondrial dysfunction, and alterations in the respiratory chain and oxidative phosphorylation systems, which when combined with the generation of the toxic free oxygen radicals as above, lead to cardiomyocyte toxicity. ${ }^{10,11}$

Cardiac function assessment and monitoring should occur prior to initiation of doxorubicin therapy. ${ }^{12}$ Although standard echocardiograms can provide a generalized cardiac function and dysfunction profile, serially performed radionuclide-based exams have been shown to provide a more sensitive assessment of cardiac function. ${ }^{13}$ Baseline radionuclide angiocardiography should be performed to calculate LVEF and then a subsequent study should be done at least three weeks after the completion of one cycle, but prior to the next dosing cycle. Patients with a normal baseline LVEF of $\geq 50 \%$ should have additional angiocardiography performed after $250-300 \mathrm{mg} / \mathrm{m}^{2}$ cumulative dose. In patients with known cardiac disease, abnormal EKG findings, a history of radiation exposure, or concurrent cyclophosphamide therapy, angiocardiography should be repeated at $400 \mathrm{mg} / \mathrm{m}^{2}$ cumulative dose. If these risk factors are not present, the study can be done at $450 \mathrm{mg} / \mathrm{m}^{2}$ cumulative dose. Additionally, doxorubicin therapy should be discontinued if the LVEF decreases below $50 \% .^{12}$

Patients with a baseline $\mathrm{LVEF} \leq 50 \%$ require closer monitoring. In patients with LVEF between $30 \%$ and $50 \%$, radionuclide angiocardiography should be performed prior to each dosing cycle. In patients with LVEF <30\%, doxorubicin should not be initiated. Additionally, patients with a baseline LVEF $\leq 50 \%$ who experience a decrease in $\mathrm{EF} \geq 10 \%$ or have a final $\mathrm{EF} \leq 30 \%$ should immediately cease doxorubicin therapy. ${ }^{12}$

Clinical manifestations of anthracycline-induced cardiomyopathy can occur within one year of initiation of chemotherapy, or up to 20 years afterwards, but the peak time to appearance of heart failure symptoms is within 3 months of completing therapy. ${ }^{4}$ Anthracycline infusion can cause an acute decline in myocardial contractility, which may lead to arrhythmias, heart failure, or subclinical EKG variations such as non-specific ST-T wave changes, however, this acute change is usually reversible. Overall, acute cardiotoxicity is a rare event, but subclinical changes may cause progressive cardiomyocyte injury leading to eventual cardiac dysfunction many years after therapy completion.

Chronic cardiotoxicity resulting from anthracycline use may not be seen until 10-20 years after therapy initiation. The most common presentation is systolic or diastolic cardiac dysfunction with progression to cardiomyopathy and clinical heart failure due to permanent and irreversible cardiomyocyte loss. The incidence of cardiotoxicity depends on the cumulative dose used, with rates of $0.14 \%$ with cumulative doses $<400 \mathrm{mg} / \mathrm{m}^{2}, 7 \%$ between $400-550$ $\mathrm{mg} / \mathrm{m}^{2}$, and $18 \%$ at doses $>700 \mathrm{mg} / \mathrm{m}^{2}$. ${ }^{4}$ Some studies have suggested cardiotoxic rates as high as $36 \%$ as cumulative doses exceed $600 \mathrm{mg} / \mathrm{m}^{2}{ }^{14}$

The prognosis of anthracycline-induced cardiotoxicity is directly related to the severity of symptoms. Some studies have suggested that carvedilol, a non-selective beta blocker, may be able to mitigate cardiac damage by protecting the mitochondrial membrane through its antioxidant properties, however, there is no clear evidence to suggest any mortality or morbidity benefit to the prophylactic use of medical therapy (such as beta blockers or ACE inhibitors) prior to initiation of anthracycline therapy. ${ }^{15}$ It is unclear if management of anthracyclineinduced cardiac dysfunction should be addressed similarly to heart failure, arrhythmias, or coronary artery disease caused by other factors.

\section{Non-anthracyclines}

Non-anthracyline agents can be broadly categorized into antimetabolites, microtubule agents, and alkylating agents. Within these classes, 5-fluorouracil (5-FU), an antimetabolite, is the most common agent associated with cardiotoxicity, and is the second most common agent overall after the anthracyclines. ${ }^{16,17}$ The reported incidence of cardiotoxicity of 5-FU varies from 1-68\% and is most commonly associated with anginal pain that occurs within 72 hours of treatment initiation, however, rare life threatening cardiac pathology such as ventricular tachycardia, cardiogenic shock, and myocardial infarction have also been reported. ${ }^{16,18,19}$

5-fluorouracil has multiple mechanisms of action but acts predominantly as a thymidylate synthase inhibitor leading to decreased DNA synthesis and repair of cancer cells. However, the pathogenic mechanism leading to cardiac injury is not fully understood and based off a limited number of studies, but thought to be due to coronary vasospasm from transient vasoconstriction. ${ }^{20,21}$ Several studies have shown that both the coronary artery and brachial artery exhibit vasospasm after 5-FU administration. ${ }^{20}$ Studies in animal models have shown a dose-related vasospasm that resolves after 5-FU is discontinued. ${ }^{21}$ Other potential causes are endothelial damage and transient stress-induced cardiomyopathies, as 
well as coronary thrombosis and arteritis, leading to manifestations such as asymptomatic EKG changes to acute pulmonary edema, arrhythmias, and myocardial infarctions with ST segment and cardiac biomarker elevations. $^{22}$

The risk of cardiotoxicity of 5-FU depends on the route and schedule of administration but is not necessarily dependent on underlying cardiovascular disease. ${ }^{19}$ Several studies in animal models have shown that 5-FU administration can lead to depletion of high energy phosphates in both a time and dosing dependent fashion which may be the etiology of angina. ${ }^{23,24}$ In addition, infusions have a higher risk of cardiotoxicity in comparison to bolus regimens but the relationship between cumulative dosage and cardiotoxicity risk is unclear. ${ }^{16,17}$ If signs and symptoms of cardiotoxicity arise, 5-FU therapy should be stopped immediately to assess whether the onset of symptoms correlate directly with the therapy involved. Anti-anginal agents should be provided for symptomatic relief. In general, re-challenging the patient should be avoided, although switching to a bolus regimen is a reasonable option.

Capecitabine, an oral fluoropyrimidine antimetabolite, has a similar cardiotoxic profiles to 5-fluorouracil. ${ }^{25}$ A recent retrospective analysis of capecitabine usage in metastatic colorectal cancer patients revealed cardiac toxicity in $5.9 \%$ of patients, with ischemia/infarction and arrhythmias the most common findings. ${ }^{26}$

Other antimetabolites include fludarabine and cytarabine. Fludarabine is not associated with cardiac toxicity as a single agent, but when combined with melphalan, an alkylating agent, can cause severe cardiac toxicity such as acute left ventricular failure and flash pulmonary edema. ${ }^{27}$ Cytarabine can lead to pericarditis, pericardial effusion, and/or pericardial tamponade, which should be treated with high-dose corticosteroids.

\section{Monoclonal antibodies to HER2}

Monoclonal antibodies against HER-2 receptors include trastuzumab and pertuzumab. HER-2 receptors are involved in gene signalling and are important in stimulating cell proliferation. In certain types of breast cancers, these genes are over-expressed, allowing cancer cells to bypass the checkpoints of normal mitotic cell division and multiply uncontrollably. Trastuzumab binds to the HER2/neu receptor to cause arrest of cells in the G1 phase of the cell cycle to restrict cellular growth and turnover.

The mechanism of trastuzumab leading to cardiotoxicity is not well understood even though it has been studied in multiple phase 3 trials. ${ }^{28-31}$ However, the cardiotoxicity risk for trastuzumab appears to be small-to-moderate compared to more toxic agents such as the anthracyclines and 5-fluorouracil. ${ }^{32}$ Anthracycline usage leads to type I cardiac dysfunction which is associated with myocyte destruction. Trastuzumab, however, is associated with type II dysfunction, which leads to myocyte "stunning" as opposed to destruction, resulting in transient changes. ${ }^{33}$ In addition, the cardiotoxicity caused by trastuzumab is not dose-dependent like other chemotherapeutic agent and is usually reversible, unlike the well-known dose-dependent cardiotoxicity seen with anthracycline usage. ${ }^{32,34}$ Thus, the manifestations of toxicity are more subtle, with asymptomatic decrease in left ventricular ejection fraction and transient EKG changes the most common presentations. ${ }^{28-30,32}$ Acute decompensated heart failure does occur, but at lower rates than other agents, which suggests that less intensive cardiac monitoring may be reasonable in patients receiving isolated trastuzumab chemotherapy. ${ }^{28-30,32}$

Pertuzumab is a newer recombinant monoclonal antibody that is used in combination with trastuzumab as neoadjuvant therapy to limit the dimerization of the HER2 receptor with other HER receptors. ${ }^{36-38}$ Dual therapy against the HER2 receptor shown to decrease disease progression and increase survival. ${ }^{36-38}$ However, the addition of pertuzumab has not been shown to increase cardiac toxicity when compared to trastuzumab therapy alone, with the majority of the events being both reversible and clinically manageable. ${ }^{36-38}$

According to the United States Food and Drug Administration guidelines, patients treated with pertuzumab are recommended to have LVEF evaluated at the beginning of treatment and every 3 months for individuals with metastatic disease and every 6 weeks for those undergoing neoadjuvant treatment. ${ }^{39}$ If the LVEF drops below $45 \%$, or is between $45-49 \%$ with a $10 \%$ or greater drop in absolute value, pertuzumab (and trastuzumab) should be held and a repeat assessment should be performed within three weeks. At this point, the clinician should take into account the benefits versus the risks for the individual before deciding to reinitiate therapy. Despite these warnings, the overall risk for cardiotoxicity remains low as described above.

\section{Tyrosine kinase inhibitors}

Tyrosine kinase is an enzyme that activates other proteins through signal transduction. Lapatinib is a tyrosine kinase inhibitor that is used in combination with other regimens (i.e. capecitabine) in breast cancer patients who are resistant to trastuzumab, targeting both the epidermal growth factor receptor (EGFR) and HER2/neu pathways by attaching to tyrosine kinases to reversibly block ATP and downstream signaling pathways. Lapatinib is usually used as a combination therapy with other HER2 agents for breast cancers that express the HER2 receptor to inhibit tumor cell proliferation and neoangiogenesis. ${ }^{40}$

Lapatinib-associated cardiotoxicity is generally reversible and non-progressive. ${ }^{41,42}$ If present, it is associated with a decrease in left ventricular ejection fraction $(\sim 20 \%)$, which can be symptomatic or asymptomatic. ${ }^{42}$ Another rare 
manifestation is QT interval prolongation, especially in patients on other QT prolonging agents. ${ }^{40}$ However, although the overall Lapatinib-related cardiotoxicity rates remain unclear, they do not appear to be dose-related.

Sunitinib is another tyrosine kinase inhibitor used for the treatment of renal cell and hepatocellular carcinomas. Studies regarding its safety profile are limited, however, one study noted modest decreases in LVEF of approximately $10 \%$, with the majority of these effects occurring in the first treatment cycle. ${ }^{43}$ Another study noted that $47 \%$ of patients developed new- onset hypertension as well. ${ }^{44}$ However, these side effects were noted to be manageable with proper monitoring of both blood pressure and LVEF changes. ${ }^{43}$

\section{Other monoclonal antibodies}

Bevacizumab is a recombinant monoclonal antibody that blocks angiogenesis by inhibiting vascular endothelial growth factor A. Although the occurrence of hypertension is a known side effect, the incidence of heart failure or cardiomyopathy is low $(<3 \%)$ in patients receiving isolated bevacizumab therapy. ${ }^{45,46}$ Given that this data is mostly collected from the adverse events of clinical trials involving breast cancer patients, the actual cardiotoxicity rates of bevacizumab remain unknown. However, combination therapy with capecitabine can increase severe cardiotoxicity risk up to $12 \% .^{26}$ Physicians should be cognizant of the increased risk of cardiotoxicity in patients receiving concurrent chemotherapy.

Funding: No funding sources Conflict of interest: None declared

Ethical approval: Not required

\section{REFERENCES}

1. Cardiotoxicity of cancer therapy. Floyd JD, Nguyen DT, Lobins RL, Bashir Q, Doll DC, Perry MC. J Clin Oncol. 2005;23(30):7685.

2. Monsuez JJ, Charniot JC, Vignat N, Artigou JY. Cardiac side-effects of cancer chemotherapy. Int $\mathrm{J}$ Cardiol. 2010;144(1):3.

3. Seidman A, Hudis C, Pierri MK, Shak S, Paton V, Ashby M, et al. Cardiac Dysfunction in the Trastuzumab Clinical Trials Experience. Journal of Clinical Oncology. 2002;20(5):1215-21.

4. Von Hoff DD, Layard MW, Basa P, Davis HL Jr, Von Hoff AL, Rozencweig M, et al. Risk factors for doxorubicin-induced congestive heart failure. Ann Intern Med. 1979;91(5):710.

5. Curigliano G, Cardinale D, Suter T, Plataniotis G, de Azambuja E, Sandri MT, et al. Cardiovascular toxicity induced by chemotherapy, targeted agents and radiotherapy: ESMO Clinical Practice Guidelines. Ann Oncol. 2012;23(7):vii155-66.

6. Steinherz LJ, Steinherz PG, Tan CT. Cardiac toxicity 4 to 20 years after completing anthracycline therapy. JAMA. 1991;266:1672-7.
7. Mimnaugh EG, Trush MA, Bhatnagar. Enhancement of reactive oxygen-dependent mitochondrial membrane lipid peroxidation by the anticancer drug adriamycin. Biochem Pharmacol. 1985;34:847-56.

8. Singal PK, Deally CMR, Weinberg LE. Subcellular effects of adriamycin in the heart: a concise review. J Mol Cell Cardiol. 1987;19:817-28.

9. Lyu YL, Kerrigan JE, Lin CP, Azarova AM, Tsai YC, Ban Y, et al. Topoisomerase II beta mediated DNA double-strand breaks: implications in doxorubicin cardiotoxicity and prevention by dexrazoxane. Cancer Res. 2007 Sept 15;67(18):8839-46.

10. Doxorubicin cardiotoxicity: Analysis of prevailing hypothesis. Olson RD, Mushlin PS. FASEB J. 1990;4:3076-86.

11. Tokarska-Schlattner M, Zaugg M, Zuppinger C. New insights into doxorubicin-induced cardiotoxicity. The critical role of cellular energetics. J Mol Cell Cardiol 2006;41:389-405.

12. Schwartz RG, McKenzie WB, Alexander J, Sager P, D'Souza A, Manatunga A, et al. Congestive heart failure and left ventricular dysfunction complicating doxorubicin therapy. Seven-year experience using serial radionuclide angiocardiography. Am J Med. 1987 Jun;82(6):1109-18.

13. Ganz WI, Sridhar KS, Ganz SS. Review of Tests for monitoring doxorubicin-induced cardiomyopathy. Oncology. 1996;53:461-70.

14. Machado V, Cabral A, Monteiro P. Carvedilol as a protector against the cardiotoxicity induced by anthracyclines (doxorubicin). Rev. Port. Cardiol. 2008;27:1277-96.

15. Feuerstein G, Yue TL, Ma X. Novel mechanisms in the treatment of heart failure: inhibition of oxygen radicals and apoptosis by carvedilol. Prog Cardiovas Dis. 1998;41:17-24.

16. Labianca R, Beretta G, Clerici M. Cardiac toxicity of 5-fluorouracil: a study on 1083 patients. Tumori. 1982;68:505

17. Anand AJ. Fluorouracil cardiotoxicity. Ann Pharmacother. 1994;28:374.

18. Yeh ET, Bickford CL. Cardiovascular complications of cancer therapy: incidence, pathogenesis, diagnosis, and management. J Am Coll Cardiol. 2009;53:223147.

19. Saif MW, Shah MM, Shah AR. Fluoropyrimidineassociated cardiotoxicity: revisited. Expert Opin Drug Saf. 2009;8(2):191.

20. Shoemaker L, Arora U, Rocha Lima C. 5-fluorouracilinduced coronary vasospasm. Cancer Control. 2004; $11: 46-9$.

21. Sudhoff T, Enderle M, Pahlke M. 5-fluorouracil induces arterial vasocontractions. Ann Oncol. 2004;15:661-4.

22. Madeddu C, Deidda M, Piras A. Pathophysiology of cardiotoxicity induced by nonanthracycline chemotherapy. Journal of Cardiovascular Medicine. 2016;17:12-218. 
23. Matsubara I, Kamiya J, Imai S. Cardiotoxic effects of 5-fluorouracil in the guinea pig. Jpn J Pharmacol. 1980;30:871-9.

24. Freeman NJ, Costanza ME. 5-Fluorouracil-associated cardiotoxicity. Cancer. 1988;61:36-45.

25. Saif MW, Quinn MG, Thomas RR, Ernst A, Grem JL. Cardiac toxicity associated with capecitabine therapy. Acta Oncol. 2003;42(4):342-4.

26. Johannes JM, Lieke HJ, Linda M. Incidence of capecitabine-related cardiotoxicity in different treatment schedules of metastatic colorectal cancer: A retrospective analysis of the CAIRO studies of the Dutch Colorectal Cancer Group. European Journal of Cancer. 2017;76:63-9.

27. Ritchie DS, Seymour JF, Roberts AW. Acute left ventricular failure following melphalan and fludarabine conditioning. Bone Marrow Transplantation. 2001;28:1:101-3.

28. Keefe DL. Trastuzumab-associated cardiotoxicity. Cancer. 2002;95(7):1592.

29. Perez EA, Rodeheffer R. Clinical cardiac tolerability of trastuzumab. J Clin Oncol. 2004;22(2):322.

30. Fiúza M. Cardiotoxicity associated with trastuzumab treatment of HER2+ breast cancer. Adv Ther. 2009;26(1):S9.

31. Martel S, Maurer C, Lambertini M. Breast cancer treatment-induced cardiotoxicity. Expert Opinion on Drug Safety. 2017;16:9:1021-38.

32. Serie DJ, Crook JE, Necela BM. Genome-wide association study of cardiotoxicity in the NCCTG N9831 (Alliance) adjuvant trastuzumab trial Pharmacogenetics and Genomics. 2017;27:378-85.

33. Ewer MS, Lippman SM. Type II chemotherapyrelated cardiac dysfunction: time to recognize a new entity. J Clin Oncol. 2005;23:2900

34. Narahara KA, Singer JW, Ritchie JL. Time- and dosedependent changes in ejection fraction after anthracycline therapy. J Cardiovasc Pharmacol. 1979;1:395-401.

35. Yu AF, Mukku RB, Verma S. Cardiac safety of nonanthracycline trastuzumab-based therapy for HER2positive breast cancer. Breast Cancer Research and Treatment. 2017;1-7.

36. Swain SM, Ewer MS, Cortes J. Cardiac tolerability of pertuzumab plus trastuzumab plus docetaxel in patients with HER2-positive metastatic breast cancer in Cleopatra: a randomized, double-blind, placebocontrolled phase III study. Oncologist. 2013;18:257.
37. Gianni L, Pienkowski T, Im YH. Efficacy and safety of neoadjuvant pertuzumab and trastuzumab in women with locally advanced, inflammatory, or early HER2-positive breast cancer (NeoSphere): a randomised multicentre, open-label, phase 2 trial. Lancet Oncol. 2012;13:25-32.

38. Von Minckwiz G, Procter M, de Azambuja E. Adjuvant pertuzumab and trastuzumab in early HER2positive breast cancer. N Engl J Med [Internet]. 2017.

39. Daily MED - PERJETA- Pertuzumab Injection, Solution Concentrate. U.S. National Library of Medicine. National Institutes of Health, 19 Dec 2017. Available at: https://dailymed.nlm.nih.gov/dailymed/drugInfo.cfm ?setid=17f85d17-ab71-4f5b-9fe3-0b8c822f69ff

40. Rosa GM, Gigli L, Tagliasacchi MI. Update on cardiotoxicity of anti-cancer treatments. European Journal of Clinical Investigation. 2016;46:3:264-84.

41. Sendur MA, Aksoy S, Altundag K. Cardiotoxicity of novel HER2-targeted therapies. Curr Med Res Opin. 2013 Aug;29(8):1015-24.

42. Perez EA, Koehler M, Byrne J, Preston AJ, Rappold E, Ewer MS. Cardiac safety of lapatinib: pooled analysis of 3689 patients enrolled in clinical trials. Mayo Clin Proc. 2008 Jun;83(6):679-86.

43. Narayan V, Keefe S, Haas N. Prospective evaluation of sunitinib-induced cardiotoxicity in patients with metastatic renal cell carcinoma. Clinical Cancer Research. 2017;23:14:3601-9.

44. Chu TF, Rupnick MA, Kerkela R. Cardiotoxicity associated with tyrosine kinase inhibitor sunitinib. Lancet. 2007;270(9604):2011-9.

45. Varga ZV, Ferdinandy P, Liaudet L, Pacher P. Druginduced mitochondrial dysfunction and cardiotoxicity. Am J Physiol Heart Circ Physiol. 2015 Nov;309(9):H1453-67.

46. Miller KD, Chap LI, Holmes FA, Cobleigh MA, Marcom PK, Fehrenbacher L, et al. Randomized phase III trial of capecitabine compared with bevacizumab plus capecitabine in patients with previously treated metastatic breast cancer. J Clin Oncol. 2005;23:792-9.

Cite this article as: Chang EY, Chaudhry AF, Lopez V, Jain SR. Cancer treatment associated cardiac toxicities. Int J Basic Clin Pharmacol 2018;7:353-8. 